\title{
Energy-efficient resource allocation for OFDMA- based two-way relay channel with physical-layer network coding
}

\author{
Min Zhou ${ }^{1 *}$, Qimei Cui ${ }^{1}$, Mikko Valkama ${ }^{2}$ and Xiaofeng Tao ${ }^{1}$
}

\begin{abstract}
Physical-layer network coding (PNC) is a novel cooperative technique for two-way relay channel (TRC), where two users exchange information via intermediate relay node(s). On the other hand, the issue of green communications to reduce energy consumption has recently started to arouse much attention. This article studies the energyefficient resource allocation problem for orthogonal frequency division multiple access (OFDMA)-based TRC with PNC. In particular, resource allocation for multi-user, multi-relay OFDMA systems is vital for the optimization of power allocation, relay selection and subcarrier assignment. By applying convex optimization techniques, an optimal resource allocation scheme is proposed to minimize total transmit power with required rates. Numerical simulations show that the proposed scheme provides diversity gain compared to the single relay network, and PNC gain relative to the TRC without PNC.
\end{abstract}

\section{Introduction}

Wireless relay was introduced in 3GPP-LTE-Advanced [1] for throughput enhancement and coverage extension without requiring large transmit powers. However, practical relay systems typically consider half-duplex mode [2], where relay nodes cannot transmit and receive simultaneously. For the purpose of overcoming the spectral loss of half-duplex relay, physical-layer network coding (PNC) [3-7] was proposed in two-way relay channel (TRC), where two users wanted to exchange information with each other via relay node(s).

Compared with the traditional one-way relay schemes that need four time slots to finish information exchange in TRC, network coding (NC) scheme [8] needs three time slots. PNC makes use of the additive nature of simultaneously arriving electromagnetic waves for equivalent network coding operation at physical layer. The number of required time slots of PNC is reduced to two: in the first time slot, two users transmit their signals simultaneously to relay node(s); then in the second time slot, relay node(s) broadcast the processed version

\footnotetext{
* Correspondence: zmzmzm82@163.com

'Wireless Technology Innovation Institute, Key Laboratory of Universal Wireless Communication, Ministry of Education, Beijing University of Posts and Telecommunications, Beijing, 100876, China

Full list of author information is available at the end of the article
}

of the received superimposed signal to the two users. Distinguished by the process function of relay node(s), PNC has several sub-protocols, such as denoise-and-forward (PNC-DNF) [3,4], decode-and-forward (PNC-DF) [5] and amplify-and-forward (PNC-AF) [5-7]. To the best of authors' knowledge, PNC-AF is the only protocol of PNC that has been demonstrated in a practical system with a bit rate of $500 \mathrm{kbps}$ [6]. Since PNC-AF is most likely to be realized in practical systems also more widely, this article will focus on PNC-AF.

OFDM/OFDMA is one of the most important transmission techniques for future wireless communication systems. In addition, TRC with PNC that employs OFDM/OFDMA has recently attracted considerable attention. Maximizing the system rate by varying the power allocation and tone permutation in TRC with single relay over OFDM was considered in [9]. Power allocation to maximize the sum-rate under the total power constraint was developed for a TRC exchanging OFDM signals via a single relay in $[10,11]$. The resource allocation problem for the OFDMA-based multiuser TRC system was investigated by [12], where an iterative algorithm was proposed to maximize the sum-rate. In [13], adaptive subcarrier allocation was proposed to maximize the achievable sum-rate for a multi-user multi-relay OFDMA-based TRC. In summary, the past 
literatures mainly focus on maximizing sum-rate to achieve full system load, which rarely happens in practical systems, even at peak traffic hours [14]. Hence, the systems optimized for full system load should be redesigned for arbitrary system load to achieve better energy efficiency.

In this article, an energy-efficient resource allocation to minimize transmit power consumption with required link rates is considered for OFDMA-based TRC with PNC. Additionally, OFDMA-based TRC indicates that the subcarriers of an OFDM symbol can be shared by multiple relay nodes. The main contributions of our study are:

- An optimal energy-efficient resource allocation is proposed with joint power allocation, relay selection and subcarrier assignment. To the best of authors' knowledge, this problem is considered for the first time in OFDMA-based TRC with PNC and multiple relay nodes.

- The best energy-efficient subcarrier assignment for OFDMA-based TRC with PNC is proved to be an opportunistic subcarrier assignment, where each subcarrier is assigned to a unique relay node.

- The closed-form expressions of optimal power allocation, relay selection, subcarrier assignment and minimum transmit power consumption are derived through convex optimization techniques.

This article is organized as follows. In Section 2, the system model is given. In Section 3, energy-efficient resource allocation problem is formulated as an optimization problem, which has been decomposed to $N$ persubcarrier problems. In Section 4, the per-subcarrier problem is solved and the joint power allocation, relay selection and subcarrier assignment scheme is proposed to achieve the minimum transmit power consumption. Section 5 gives numerical simulations and performance analysis under the energy efficiency evaluation framework $\left(\mathrm{E}^{3} \mathrm{~F}\right)$ provided in EARTH project [15]. Conclusions are given in the last section.

Notation: $[\bullet]^{T}$ denotes the transpose of vector. $|\bullet|$ denotes the scalar norm. For vectors, $\geqslant$ and $=$ are used to indicate the componentwise inequality and equality, respectively.

\section{System model}

This article considers a TRC with multiple relay nodes as shown in Figure 1, which has two users, $K$ halfduplex relay nodes and $N$ subcarriers. Suppose that users $S_{1}$ and $S_{2}$ intend to exchange information with each other and there is no direct path between the two users. Hence, the exchange will be finished with the assistance of the relay nodes. It is assumed that all the nodes are equipped with a single antenna and operated in a time division duplexing (TDD) mode. Assuming channel reciprocity, which is justified by the TDD mode, given that the duplexing period is small compared to channel coherence time, the complex channel coefficient from $S_{i}$ to $R_{i}$ is same as channel from $R_{i}$ to $S_{i}$. Since OFDM divides the wideband signal into many narrowband subcarriers, with proper cyclic prefixing, the individual subcarriers in an OFDM signal experience frequency-flat fading. For generality, we also assume that the subcarrier responses depend in the general case on subcarrier index which is denoted here by $n$. The amount of correlation between different subcarriers depends on the relation of channel coherence bandwidth and subcarrier spacing. In terms of mobility and Doppler spread, we assume that the channel coherence time is long compared to OFDM symbol duration. Accordingly, this article considers a quasi-static fading channel for which the channels are constant within one frame but change independently from one frame to another. Furthermore, we also assume that the radio channels corresponding to different relay nodes are

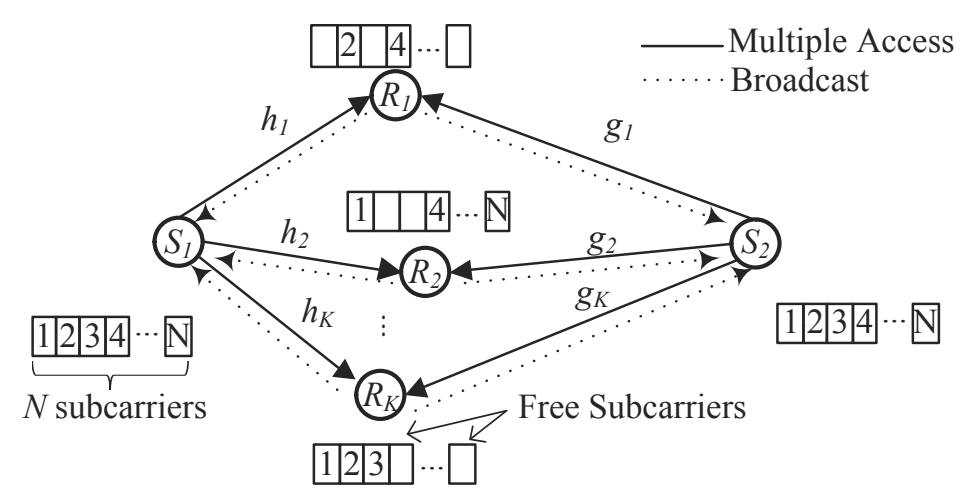

Figure 1 OFDMA-based TRC with $K$ relay nodes and $N$ subcarriers. 
mutually independent. In addition, the complex channel coefficient on the $n$th subcarrier between $S_{1}$ and $R_{i}$, and $S_{2}$ and $R_{i}$ are denoted by $h_{i}^{(n)}$ and $g_{i}^{(n)}$, respectively, while the Additive White Gaussian Noise (AWGN) at $S_{1}, S_{2}$, and $R_{i}$ on the $n$th subcarrier are denoted by $u_{i}^{(n)}$, and $u_{i}^{(n)}$, respectively.

The considered PNC-AF protocol finishes the information exchange in two time slots. In the first time slot for multiple access phase, user $S_{1}$ transmit data symbol $x_{1}^{(n)}$ with power $P_{s, 1}^{(n)}$ on the $n$th subcarrier, while user $S_{2}$ transmit $x_{2}^{(n)}$ with power $P_{s, 2}^{(n)}$. Assuming perfect synchronization, the received signal at relay node $R_{i}$ on $n$th subcarrier is expressed as:

$$
y_{i}^{(n)}=h_{i}^{(n)} \sqrt{P_{s, 1}^{(n)}} x_{1}^{(n)}+g_{i}^{(n)} \sqrt{P_{s, 2}^{(n)}} x_{2}^{(n)}+u_{i}^{(n)}
$$

Then, if the relay node $R_{i}$ is selected to participate in transmission and assigned with the $n$th subcarrier, $R_{i}$ will amplify the received signal on the $n$th subcarrier by multiplying $y_{i}^{(n)}$ with amplification factor $\sqrt{\alpha_{i}^{(n)}}$. To make the transmitted power at $R_{i}$ on the $n$th subcarrier equal to $P_{r, i}^{(n)}$, the factor $\alpha_{i}^{(n)}$ is expressed as

$$
\alpha_{i}^{(n)}=\frac{P_{r, i}^{(n)}}{\left|h_{i}^{(n)}\right|^{2} P_{s, 1}^{(n)}+\left|g_{i}^{(n)}\right|^{2} P_{s, 2}^{(n)}+N_{0} W}
$$

where $N_{0}$ is power spectral density of AWGN and $W$ denotes the subcarrier bandwidth.

Let $\tau_{i}$ and $\rho_{i}^{\left({ }^{(n)}\right.}$ be the relay selection indicator variable and subcarrier assignment indicator variable on $n$th subcarrier, respectively, where

$$
\tau_{i}=\left\{\begin{array}{l}
1, \text { when } R_{i} \text { is selected to patriciate in transmission } \\
0, \text { otherwise }
\end{array}\right.
$$

and

$$
\rho_{i}^{(n)}=\left\{\begin{array}{l}
1, \text { when the } n \text {th subcarrier is assigned to } R_{i} \\
0, \text { otherwise }
\end{array}\right.
$$

By taking $\rho_{i}^{(n)}$ and $\tau_{i}$ into account, we use $\sqrt{\tau_{i} \rho_{i}^{(n)} \alpha_{i}^{(n)}}$ as a general scale factor for $R_{i}$ on $n$th subcarrier. It is noted that the $n$th subcarrier of relay node $R_{i}$ is marked as free subcarrier (as shown in Figure 1), while $\rho_{i}^{(n)}$ is equal to zero.

In the second time slot for broadcast phase, all the relay nodes broadcast the scaled signals to the two users. The received signals at $S_{1}$ and $S_{2}$ on the $n$th subcarrier are given, respectively, by

$$
\tilde{z}_{1}^{(n)}=\sum_{i=1}^{K} h_{i}^{(n)} \sqrt{\tau_{i} \rho_{i}^{(n)} \alpha_{i}^{(n)}} \gamma_{i}^{(n)}+v_{1}^{(n)}
$$

and

$$
\tilde{z}_{2}^{(n)}=\sum_{i=1}^{K} g_{i}^{(n)} \sqrt{\tau_{i} \rho_{i}^{(n)} \alpha_{i}^{(n)}} \gamma_{i}^{(n)}+v_{2}^{(n)}
$$

Assuming that channel state information (CSI) is available, each user can cancel its own transmit signal component from the received signal. Therefore, the resulting signals at $S_{1}$ and $S_{2}$ on the $n$th subcarrier can be written as

$$
z_{1}^{(n)}=\sum_{i=1}^{K} h_{i}^{(n)} \sqrt{\tau_{i} \rho_{i}^{(n)} \alpha_{i}^{(n)}}\left(g_{i}^{(n)} \sqrt{P_{s, 2}^{(n)}} x_{2}^{(n)}+u_{i}^{(n)}\right)+v_{1}^{(n)}(7)
$$

and

$$
z_{2}^{(n)}=\sum_{i=1}^{K} g_{i}^{(n)} \sqrt{\tau_{i} \rho_{i}^{(n)} \alpha_{i}^{(n)}}\left(h_{i}^{(n)} \sqrt{P_{s, 1}^{(n)}} x_{1}^{(n)}+u_{i}^{(n)}\right)+v_{2}^{(n)}(8)
$$

\section{Problem formulation and simplification}

In this section, the optimal energy-efficient resource allocation is formulated as a joint optimization of power allocation, relay selection and subcarrier assignment to achieve the minimum transmit power consumption with required link rates. With the mathematical analysis, the optimization problem has been decomposed into $N$ subproblems. Then, the subproblems are simplified by using the Karush-Kuhn-Tucker (KKT) condition.

\subsection{Problem formulation}

The objective is to find the optimal subcarrier assignment $\left\{\rho_{i}^{(n)}\right\}$, relay selection $\left\{\tau_{i}\right\}$ and power allocation $\left\{P_{s, 1}^{(n)}, P_{s, 2}^{(n)}, P_{r, i}^{(n)}\right\}$ that minimize the overall transmit power subject to satisfying each link's rate requirement $\bar{r}_{1}$ and $\bar{r}_{2}$ in bit/s for links $S_{1} \rightarrow$ relay(s) $\rightarrow S_{2}$ and $S_{2} \rightarrow$ relay (s) $\rightarrow S_{1}$, respectively. The optimization is formulated as (Problem 3.1):

$$
\begin{aligned}
& \underset{P_{s, 1}^{(n)}, P_{s, 2}^{(n)}, P_{r, i}^{(n)}, \rho_{i}^{(n)}, \tau_{i}, \forall i}{\operatorname{minimize}} \sum_{n=1}^{N}\left(P_{s, 1}^{(n)}+P_{s, 2}^{(n)}+\sum_{i=1}^{K} \tau_{i} \rho_{i}^{(n)} P_{r, i}^{(n)}\right) \\
& \text { subject to } \sum_{n=1}^{N} r_{1}^{(n)} \geq \bar{r}_{1}, \sum_{n=1}^{N} r_{2}^{(n)} \geq \bar{r}_{2} \\
& P_{s, 1}^{(n)} \geq 0, P_{s, 2}^{(n)} \geq 0, P_{r, i}^{(n)} \geq 0 \forall i, n
\end{aligned}
$$




$$
\rho_{i}^{(n)} \in\{0,1\}, \tau_{i} \in\{0,1\} \forall i, n
$$

where $r_{1}^{(n)}$ and $r_{2}^{(n)}$ are the achievable rates on the $n$th subcarrier for links $S_{1} \rightarrow$ relay(s) $\rightarrow S_{2}$ and $S_{2} \rightarrow$ relay $(\mathrm{s}) \rightarrow S_{1}$, respectively. From (7) and (8), the expressions of $r_{1}^{(n)}$ and $r_{2}^{(n)}$ are given by

$$
r_{1}^{(n)}=\frac{1}{2} W \log _{2}\left(1+\frac{\sum_{i=1}^{K} \tau_{i} \rho_{i}^{(n)}\left|g_{i}^{(n)}\right|^{2}\left|h_{i}^{(n)}\right|^{2} \alpha_{i}^{(n)} P_{s, 1}^{(n)}}{\left(1+\sum_{i=1}^{K} \tau_{i} \rho_{i}^{(n)}\left|g_{i}^{(n)}\right|^{2} \alpha_{i}^{(n)}\right) N_{0} W}\right)
$$

and

$$
r_{2}^{(n)}=\frac{1}{2} W \log _{2}\left(1+\frac{\sum_{i=1}^{K} \tau_{i} \rho_{i}^{(n)}\left|g_{i}^{(n)}\right|^{2}\left|h_{i}^{(n)}\right|^{2} \alpha_{i}^{(n)} P_{s, 2}^{(n)}}{\left(1+\sum_{i=1}^{K} \tau_{i} \rho_{i}^{(n)}\left|h_{i}^{(n)}\right|^{2} \alpha_{i}^{(n)}\right) N_{0} W}\right)
$$

The factor $1 / 2$ comes from the two time slots required by messages exchange. However, Problem 3.1 has high computational complexity, while optimum values of $K$ $(2 N+1)+2 N$ variables need to be found. Some simplifications will be implemented in the following section.

\subsection{Problem simplification}

Equal rate allocation is considered in this article to simplify the complexity of problem. On average, the rate of each subcarrier is $\frac{\bar{r}_{1}}{N}$ bps for link $S_{1} \rightarrow$ relay(s) $\rightarrow S_{2}$ and $\frac{\bar{r}_{2}}{N}$ bps in the reverse direction. Since the separate rate requirement on each subcarrier is known, Problem 3.1 is decomposed to $N$ independent subproblems to find the minimum transmit power consumption on per-subcarrier basis. The optimization problem on the $n$th subcarrier can be expressed now as (Problem 3.2):

$$
\begin{aligned}
& \underset{P_{s, 1}^{(n)}, P_{s, 2}^{(n)}, P_{r, i}^{(n)}, \rho_{i}^{(n)}, \tau_{i}, \forall i}{\operatorname{minimize}} P_{s, 1}^{(n)}+P_{s, 2}^{(n)}+\sum_{i=1}^{K} \tau_{i} \rho_{i}^{(n)} P_{r, i}^{(n)} \\
& \text { subject to } r_{1}^{(n)} \geq \frac{\bar{r}_{1}}{N}, r_{2}^{(n)} \geq \frac{\bar{r}_{2}}{N} \\
& P_{s, 1}^{(n)} \geq 0, P_{s, 2}^{(n)} \geq 0, P_{r, i}^{(n)} \geq 0 \forall i \\
& \rho_{i}^{(n)} \in\{0,1\}, \tau_{i} \in\{0,1\} \forall i
\end{aligned}
$$

The overall transmit power consumption will be the sum of individual transmit power consumptions on each subcarrier.

In mathematics, Problem 3.2 is a nonlinear programming, where some of the constraints or the objective function are nonlinear. Karush-Kuhn-Tucker (KKT) conditions are necessary and sufficient for a solution in nonlinear programming to be optimal [16]. By substituting (2), (13), and (14) into Problem 3.2 and applying the KKT optimality conditions, the transmit power of $S_{1}$ and $S_{2}$ on the $n$th subcarrier can be expressed as:

$$
P_{s, 1}^{(n)}=\frac{\left(1+\sum_{i=1}^{K} \tau_{i} \rho_{i}^{(n)} \alpha_{i}^{(n)}\left|g_{i}^{(n)}\right|^{2}\right)\left(2 \frac{2 \bar{r}_{1}}{W N}-1\right) N_{0} W}{\sum_{i=1}^{K} \tau_{i} \rho_{i}^{(n)} \alpha_{i}^{(n)}\left|g_{i}^{(n)}\right|^{2}\left|h_{i}^{(n)}\right|^{2}}
$$

and

$$
P_{s, 2}^{(n)}=\frac{\left(1+\sum_{i=1}^{K} \tau_{i} \rho_{i}^{(n)} \alpha_{i}^{(n)}\left|h_{i}^{(n)}\right|^{2}\right)\left(2 \frac{2 \bar{r}_{2}}{W N}-1\right) N_{0} W}{\sum_{i=1}^{K} \tau_{i} \rho_{i}^{(n)} \alpha_{i}^{(n)}\left|g_{i}^{(n)}\right|^{2}\left|h_{i}^{(n)}\right|^{2}}
$$

Observation 1: It is observed that at least one subcarrier should be assigned to relay node $R_{i}$, if $R_{i}$ is selected. Hence the relay selection indicator variable can be expressed as

$$
\tau_{i}=\operatorname{sgn}\left(\sum_{n=1}^{N} \rho_{i}^{(n)}\right)
$$

where $\operatorname{sgn}(x)=\left\{\begin{array}{l}1 \forall x>0 \\ 0 \forall x \leq 0\end{array}\right.$.

Hence, it is shown that $\rho_{i}^{(n)}=\tau_{i} \rho_{i}^{(n)}$ for the per-subcarrier optimization on the $n$th subcarrier.

By substituting (2), (19), and (20) into Problem 3.2 and using Observation 1, the optimization Problem 3.2 is reduced to the following problem formulated as (Problem 3.3):

$$
\begin{aligned}
& \underset{\alpha_{i}^{(n)}, \rho_{i}^{(n)}, \forall i}{\operatorname{minimize}} \bar{P}^{(n)}\left(\alpha^{(n)}, \rho^{(n)}\right) \\
& \text { subject to } \alpha^{(n)} \succeq 0, \rho^{(n)} \in\{0,1\}
\end{aligned}
$$

where

$$
\alpha^{(n)}=\left[\alpha_{1}^{(n)}, \alpha_{2}^{(n)}, \ldots, \alpha_{K}^{(n)}\right], \rho^{(n)}=\left[\rho_{1}^{(n)}, \rho_{2}^{(n)}, \ldots, \rho_{K}^{(n)}\right] \text {. }
$$

The expression of $\bar{P}^{(n)}\left(\alpha^{(n)}, \rho^{(n)}\right)$ is given by

$$
\bar{P}^{(n)}\left(\alpha^{(n)}, \rho^{(n)}\right)=\frac{m\left(1+\sum_{i=1}^{K} \rho_{i}^{(n)} \alpha_{i}^{(n)}\left|g_{i}^{(n)}\right|^{2}\right)\left(1+\sum_{i=1}^{K} \rho_{i}^{(n)} \alpha_{i}^{(n)}\left|h_{i}^{(n)}\right|^{2}\right)}{\left(\left.\sum_{i=1}^{K} \rho_{i}^{(n)} \alpha_{i}^{(n)}\left|g_{i}^{(n)}\right|\right|^{2}\left|h_{i}^{(n)}\right|^{2}\right)}+N_{0} W \sum_{i=1}^{K} \rho_{i}^{(n)} \alpha_{i}^{(n)}
$$

where $m=\left(2 \frac{2 \bar{r}_{1}}{W N}+2 \frac{2 \bar{r}_{2}}{W N}-2\right) N_{0} W$. 
Finally, the original Problem 3.1 with $K(2 N+1)+2 N$ variables is reduced to a per-subcarrier optimization Problem 3.3 with only $2 K$ variables. Furthermore, Problem 3.3 has very simple constraints, where the only requirements are that the factors $\alpha_{i}^{(n)}$ should be nonnegative and $\rho_{i}^{(n)} \in\{0,1\}$.

Remark 1: Problem 3.3 is to find the minimum power consumption on the $n$th subcarrier. If Problem 3.3 is solved, the minimum power consumption on the other subcarriers can also be obtained with the similar steps. Accordingly, the overall minimum power consumption will be the sum of transmit power consumptions on each subcarrier. In the following section, more detailed mathematical analysis will be carried out around the per-subcarrier optimization Problem 3.3.

\section{Per-subcarrier optimization}

Problem 3.3 is a mixed binary integer programming problem, which considers the minimum power consumption on the $n$th subcarrier. However, it is prohibitive to find the global optimum in terms of computational complexity. To obtain the global optimum, an exhaustive search is needed throughout the subcarrier assignment vectors $\rho^{(n)}$ and amplification factor vectors $\alpha^{(n)}$ to find the overall minimum transmit power. Before solving the Problem 3.3 with optimal subcarrier assignment vector $\rho^{(n)}$, we first propose an energy-efficient resource allocation scheme with opportunistic subcarrier assignment in 4.1. Then, the proposed heuristic scheme is proved to be the optimal solution of Problem 3.3 in Section 4.2 .

\subsection{Energy-efficient resource allocation with opportunistic subcarrier assignment}

This section considers the Problem 3.3 with an opportunistic subcarrier assignment, where each subcarrier is assigned to an unique relay node. Hence, Problem 3.3 is divided into a power allocation and a opportunistic subcarrier assignment problem.

Power allocation criterion: It is assumed that the $n$th subcarrier is only assigned to relay node $R_{d}$. Under this assumption, we can determine the subcarrier assignments $\rho^{(n)}$, which are directly given by

$$
\rho_{i}^{(n)}=\left\{\begin{array}{l}
1 \forall i=d \\
0 \forall i \neq d
\end{array}\right.
$$

By substituting (25) into Problem 3.3, the problem becomes easy to solve. Hence, the solution is given directly as:

$$
\bar{\alpha}_{d}^{(n)}=\sqrt{\frac{m}{\left|g_{d}^{(n)}\right|^{2}\left|h_{d}^{(n)}\right|^{2}\left(m+N_{0} W\right)}}
$$

$$
\bar{P}^{(n)}(d)=\frac{m}{\left|g_{d}^{(n)}\right|^{2}}+\frac{m}{\left|h_{d}^{(n)}\right|^{2}}+2 \sqrt{\frac{m\left(m+N_{0} W\right)}{\left|g_{d}^{(n)}\right|^{2}\left|h_{d}^{(n)}\right|^{2}}}
$$

where $\bar{\alpha}_{d}^{(n)}$ is the optimum point to achieve the minimum transmit power consumption $\bar{P}^{(n)}(d)$ on $n$th subcarrier, while it is only assigned to relay node $R_{d}$. By substituting $\bar{\alpha}_{d}^{(n)}$ and (25) into (9), (19), and (20), the closed-form expression of power allocated to two users and relay node $R_{d}$ on $n$th subcarrier can be expressed as:

$$
\bar{P}_{s, 1}^{(n)}=\frac{\left(1+\bar{\alpha}_{d}^{(n)}\left|g_{d}^{(n)}\right|^{2}\right)\left(2 \frac{2 \bar{r}_{1}}{W N}-1\right) N_{0} W}{\bar{\alpha}_{d}^{(n)}\left|g_{d}^{(n)}\right|^{2}\left|h_{d}^{(n)}\right|^{2}}
$$

$$
\begin{aligned}
\bar{P}_{s, 2}^{(n)} & =\frac{\left(1+\bar{\alpha}_{d}^{(n)}\left|h_{d}^{(n)}\right|^{2}\right)\left(2 \frac{2 \bar{r}_{2}}{W N}-1\right) N_{0} W}{\bar{\alpha}_{d}^{(n)}\left|g_{d}^{(n)}\right|^{2}\left|h_{d}^{(n)}\right|^{2}} \\
\bar{P}_{r, d}^{(n)} & =\bar{\alpha}_{d}^{(n)}\left(\left|h_{d}^{(n)}\right|^{2} \bar{P}_{s, 1}^{(n)}+\left|g_{d}^{(n)}\right|^{2} \bar{P}_{s, 2}^{(n)}+1\right)
\end{aligned}
$$

Opportunistic subcarrier assignment criterion: The index $\tilde{d}$ of the relay node assigned with the $n$th subcarrier is determined as

$$
\tilde{d}=\arg \min _{d=1,2, \ldots, K}\left\{\bar{P}^{(n)}(d)\right\}
$$

Remark 2: To summarize shortly, the energy-efficient resource allocation algorithm on the $n$th subcarrier with opportunistic subcarrier assignment approach proposed above has two steps: First, the $n$th subcarrier is assigned to the best relay $R_{\tilde{d}}$ by opportunistic subcarrier assignment criterion. Then, optimal transmit power determined by power allocation criterion will be allocated to $S_{1}, S_{2}$, and $R_{\tilde{d}}$ on the $n$th subcarrier. The minimum transmit power consumption $P_{0}^{(n)}$ on the $n$th subcarrier of this scheme is given by $P_{o}^{(n)}=\bar{P}^{(n)}(\tilde{d})$.

\subsection{Energy-efficient resource allocation with optimal subcarrier assignment}

In this section, it is proved that the above opportunistic two-step resource allocation algorithm is also the optimum method in terms of energy-efficiency. This is formulated with the following Theorem:

Theorem 1 In multi-relay OFDMA-based TRC with PNC, the opportunistic subcarrier assignment is the 
optimal energy-efficient subcarrier assignment scheme, in which the nth subcarrier is assigned to the best relay node from the $K$ candidate relay nodes.

Proof: Two steps are needed to complete the proof. We first consider the necessary and sufficient conditions of Theorem 1, and then solve its corresponding mathematical problem.

Step 1: Necessary and sufficient conditions of Theorem 1: If opportunistic subcarrier assignment is the optimal scheme, the minimum transmit power consumption on the $n$th subcarrier is $P_{0}^{(n)}$, which is given in Section 4.1. Since $\bar{P}^{(n)}\left(\alpha^{(n)}, \rho^{(n)}\right)$ is the transmit power consumption on the $n$th subcarrier, Theorem 1 will be proved if we can prove that $\bar{P}^{(n)}\left(\alpha^{(n)}, \rho^{(n)}\right)$ cannot have a value less than $P_{o}^{(n)}$ in the feasible region. The mathematical expressions of the necessary and sufficient conditions are

$$
\begin{aligned}
& \bar{P}^{(n)}\left(\alpha^{(n)}, \rho^{(n)}\right)-P_{o}^{(n)} \geq 0 \\
& \alpha^{(n)} \succeq 0, \rho^{(n)} \in\{0,1\}
\end{aligned}
$$

Thus to prove Theorem 1, we start by showing that the left-hand side of (32) is positive. Let $\Xi_{1 \times K}=\underbrace{\left[\zeta_{1}, \zeta_{2}, \ldots, \zeta_{K}\right]}_{K}$, where $\zeta_{k}=\rho_{i}^{(n)} \alpha_{i}^{(n)}$. Since $\bar{P}^{(n)}\left(\alpha^{(n)}, \rho^{(n)}\right)$ has positive denominator, proof of (32) transforms to solving the following optimization problem

$$
\text { minimize } f\left(\Xi_{1 \times K}\right)
$$

$$
\text { subject to } \Xi_{1 \times K} \succeq 0
$$

where $f\left(\Xi_{1 \times K}\right)$ is the left-hand side of (32) multiplied by its denominator and is expressed in more details by (35).

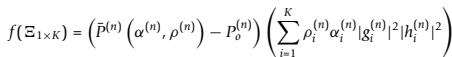

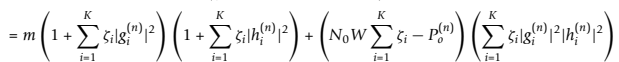

Hence, the necessary and sufficient condition of Theorem 1 is that the minimum value of $f\left(\Xi_{1 \times K}\right)$ is nonnegative in the feasible region.

Step 2: Solution of the corresponding optimization problem of necessary and sufficient conditions: Let $\gamma=P_{o}^{(n)} / N_{0} W$. It is obvious that $f\left(\Xi_{1 \times K}\right)$ is strictly increasing with $\zeta_{i}$, when $\zeta_{i}>\gamma$. So the minimum value of $f$ $\left(\Xi_{1 \times K}\right)$ is not changed while we change the constrained condition $\Xi_{1 \times K} \geqslant 0$ to $\gamma \geqslant \Xi_{1 \times K} \geqslant 0$. So the corresponding mathematical problem can be rewritten as:

$$
\text { minimize } f\left(\Xi_{1 \times K}\right)
$$

$$
\text { subject to } \gamma \succeq \Xi_{1 \times K} \succeq 0
$$

Since $\Lambda^{K}=\left\{\gamma \geqslant \Xi_{1 \times K} \geqslant 0\right\}$ is a closed and bounded set and $f\left(\Xi_{1 \times K}\right): \Lambda^{K} \rightarrow \mathbb{R}$ is continuous, problem (36) has a solution [[17], Theorem 0.3]. We consider here the second-order necessary conditions for optimality [[18], Chapter 11.5] of problem (36). The Hessian matrix of $f\left(\Xi_{1 \times K}\right)$ is

$$
\nabla^{2} f\left(\Xi_{1 \times K}\right)=\left[\begin{array}{cccc}
\psi(1) & \vartheta(1,2) & \cdots & \vartheta(1, K) \\
\vartheta(2,1) & \psi(2) & \cdots & \vartheta(2, K) \\
\vdots & \vdots & \ddots & \vdots \\
\vartheta(K, 1) & \vartheta(K, 2) & \cdots & \psi(K)
\end{array}\right]
$$

where

$$
\psi(i)=2\left|g_{i}^{(n)}\right|^{2}\left|h_{i}^{(n)}\right|^{2}\left(\mathrm{~m}+N_{0} W\right) \quad \text { and }
$$

$\vartheta(i, j)=\vartheta(j, i)=\left.\left|g_{i}^{(n)}\right|\right|^{2}\left|h_{i}^{(n)}\right|{ }^{2} N_{0} W+\left|g_{j}\right|^{2}\left|h_{j}\right|^{2} N_{0} W+\left|g_{i}^{(n)}\right|^{2}\left|h_{j}\right|^{2} \mathrm{~m}+\left|g_{j}\right|^{2}\left|h_{i}^{(n)}\right|^{2} \mathrm{~m}$.

The first-order leading principal minors of $f\left(\Xi_{1 \times K}\right)$ is $\psi(1)$, which is positive. The second-order leading principal minors of $f\left(\Xi_{1 \times K}\right)$ is that:

$$
\left|\nabla^{2} f\left(\Xi_{1 \times K}\right)_{2 \times 2}\right|=\psi(1) \psi(2)-\vartheta(1,2) \vartheta(2,1) \leq 0
$$

According to the Sylvester's criterion [[18], Theorem 7.2.5], $\nabla^{2} f\left(\Xi_{1 \times K}\right)$ must be positive semi-definite matrix (PSM) or indefinite matrix (IM). Next, we will prove that minimum value of $f\left(\Xi_{1 \times K}\right)$ is nonnegative in cases where $\nabla^{2} f\left(\Xi_{1 \times K}\right)$ is either PSM or IM.

(1) $\nabla^{2} f\left(\Xi_{1 \times K}\right)$ is PSM: It means all the second-order principal minors of $\nabla^{2} f\left(\Xi_{1 \times K}\right)$ must be zero. So, it is derived that $g_{1}^{(n)}=g_{2}^{(n)}=\cdots=g_{k}^{(n)}=h_{1}^{(n)}=h_{2}^{(n)}=\cdots=h_{k}^{(n)}$. In this condition, it is obvious that

$$
f_{\min }\left(\Xi_{1 \times K}\right)=0 \text {, while } \nabla^{2} f\left(\Xi_{1 \times K}\right) \text { is PSM }
$$

where $f_{\min }\left(\Xi_{1 \times K}\right)$ is the minimum value of function $f$ $\left(\Xi_{1 \times K}\right)$ in the feasible region.

(2) $\nabla^{2} f\left(\Xi_{1 \times K}\right)$ is IM: According to [[20], Section III], function (25) has no extreme points, in other words the minimum value must be obtained on the domain boundary (one of the $\zeta_{i}$ equals to 0 or $\gamma$ ). The values on boundary are expressed as $b_{\gamma}(w)=f_{\min }\left(\zeta_{1}, \ldots, \zeta_{w-}\right.$ $\left.{ }_{1}, \zeta_{w+1}, \ldots, \zeta_{K}\right)$ and $b_{0}(w)=f_{\min }\left(\zeta_{1}, \ldots, \zeta_{w-1}, 0, \zeta_{w+1}\right.$, $\left.\ldots, \zeta_{K}\right)$. Hence, the minimum value of $f\left(\Xi_{1 \times K}\right)$ is $f_{\min }\left(\Xi_{1 \times K}\right)=\min \left(b_{\gamma}(1), b_{\gamma}(2), \ldots, b_{\gamma}(K), b_{0}(1), b_{0}\right.$ $\left.(2), \ldots, b_{0}(K)\right)$. If we can prove that $b_{\gamma}(w) \geq 0$ and $b_{0}(w) \geq 0, \forall w=1,2, \ldots, K$, the minimum value of function $f\left(\Xi_{1 \times K}\right)$ will be positive. We can easily prove that

$$
\begin{aligned}
b_{\gamma}(w) & =f(0, \ldots, 0, \gamma, 0, \ldots, 0) \\
& =m\left(1+\gamma\left|g_{w}^{(n)}\right|^{2}\right)\left(1+\gamma\left|h_{w}^{(n)}\right|^{2}\right)>0
\end{aligned}
$$


Next, we will prove $b_{0}(w) \geq 0$. It is first known that

$$
\begin{aligned}
b_{0}(K) & =f\left(\zeta_{1}, \ldots, \zeta_{K-1}, 0\right) \\
& =m\left(1+\sum_{i=1}^{K-1} \zeta_{i}\left|g_{i}^{(n)}\right|^{2}\right)\left(1+\sum_{i=1}^{K-1} \zeta_{i}\left|h_{i}^{(n)}\right|^{2}\right) \\
& +\left(N_{0} W \sum_{i=1}^{K-1} \zeta_{i}-P_{o}^{(n)}\right)\left(\sum_{i=1}^{K-1} \zeta_{i}\left|g_{i}^{(n)}\right|^{2}\left|h_{i}^{(n)}\right|^{2}\right) \\
& =f\left(\Xi_{1 \times(K-1)}\right)
\end{aligned}
$$

If we can now prove that $b_{0}(K)=f\left(\Xi_{1 \times(K-1)}\right) \geq 0$, it can be extended to prove $b_{0}(w) \geq 0, \forall w=\{1,2, \ldots, K\}$ straightforwardly. Then, the problem degenerates to prove $f\left(\Xi_{1 \times(K-1)}\right) \geq 0$.

With the similar discussion repeated from step 2 and $K-2$ times iteration, the problem will degenerate to prove $f\left(\Xi_{1} \times{ }_{1}\right)=f\left(\zeta_{i}\right) \geq 0$. We know that $f\left(\zeta_{i}\right)=m\left(\left|g_{i}^{(n)}\right|^{2} \zeta_{i}+1\right)\left(\left|h_{i}^{(n)}\right|^{2} \zeta_{i}+1\right)+\left(N_{0} W \zeta_{i}-P_{o}^{(n)}\right)\left|g_{i}^{(n)}\right|^{2}\left|h_{i}^{(n)}\right|^{2} \zeta_{i}$ The minimum value of $f\left(\zeta_{i}\right)$ is denoted by $f_{\min }\left(\zeta_{i}\right)$, which is given by (43).

$$
\begin{aligned}
& f_{\min }\left(\zeta_{i}\right)=f\left(\frac{\left.P_{0}^{(n)}\left|g_{i}^{(n)}\right||| h_{i}^{(n)}\right|^{2}-m\left|g_{i}^{(n)}\right|^{2}-m\left|h_{i}^{(n)}\right|^{2}}{\left.2\left(m+N_{0} W\right)\left|g_{i}^{(n)}\right| h_{i}^{(n)}\right|^{2}}\right) \\
& =\frac{\left|h_{i}^{(n)}\right|^{2} m\left(\left.2\left|g_{i}^{(n)}\right|\right|^{\mid}\left|h_{i}^{(n)}\right| P_{0}^{(n)}+2\left|g_{i}^{(n)}\right|^{2} m+4\left|g_{i}^{(n)}\right|^{2} N_{0} W-\left|h_{i}^{(n)}\right|^{2} m\right)-\left|g_{i}^{(n)}\right|^{4}\left(\left|h_{i}^{(n)}\right|^{(n)} P_{0}^{(n)}-m\right)^{2}}{4\left|g_{i}^{(n)}\right|\left|h_{i}^{(n)}\right|^{2}\left(m+N_{0} W\right)}
\end{aligned}
$$

Next, we will prove that $f_{\min }\left(\zeta_{i}\right) \geq 0$. Assume first that

$$
\varphi\left(P_{o}^{(n)}\right)=4\left|g_{i}^{(n)}\right|^{2}\left|h_{i}^{(n)}\right|^{2}\left(m+N_{0} W\right) f_{\min }\left(\zeta_{i}\right)=0
$$

The two real roots of (44) are

$$
\varepsilon_{1,2}=\frac{m}{\left|g_{i}^{(n)}\right|^{2}}+\frac{m}{\left|h_{i}^{(n)}\right|^{2}} \pm 2 \sqrt{\frac{m\left(m+N_{0} W\right)}{\left|g_{i}^{(n)}\right|^{2}\left|h_{i}^{(n)}\right|^{2}}}
$$

From the definition of $P_{o}^{(n)}$, we have $\varepsilon_{2} \leq P_{o}^{(n)} \leq \varepsilon_{1}$, which implies $\varphi\left(P_{o}^{(n)}\right) \geq 0$ and then $f_{\min }\left(\zeta_{i}\right) \geq 0$. Finally, it is known that $b_{0}(K)=f\left(\Xi_{1 \times(K-1)}\right) \geq 0$, which can be extended to prove $b_{0}(w) \geq 0, \forall w=1,2, \ldots, K$ straightforwardly.

Since $b_{\gamma}(w) \geq 0$ and $b_{0}(w) \geq 0, \forall w=\{1,2, \ldots, K\}$ are proved, the following result has been achieved

$$
f_{\min }\left(\Xi_{1 \times K}\right) \geq 0 \text {, while } \nabla^{2} f\left(\Xi_{1 \times K}\right) \text { is IM }
$$

From (40) and (46), it is then clear that the minimum value of $f\left(\Xi_{1 \times K}\right)$ is nonnegative. The necessary and sufficient conditions of Theorem 1 discussed in step 1 are thus satisfied. Theorem 1 is proved.

From Theorem 1, it is known that the proposed opportunistic subcarrier assignment criterion is the optimal energy-efficient criterion in multi-user multi-relay OFDMA-based TRC with PNC-AF.

Remark 3: The overall optimal energy-efficient resource allocation algorithm is described in the Figure
2. As discussed in Section 3.2, resource allocation has been decomposed into $N$ independent subproblems on the subcarriers. Then, the per-subcarrier resource allocation is implemented from the subcarrier 1 to $N$. Finally, the subcarrier assignment, relay selection indicator variables are calculated and the minimum power consumption is achieved.

\section{Performance analysis}

To capture the energy efficiency perspective in the analysis, we employ the energy consumption index (ECI) from EARTH project [15]. ECI provides the energy per bit, which is defined as the energy consumption (E) during the observation period $(\mathrm{T})$ divided by the total



Figure 2 Flow chart of resource allocation algorithm 


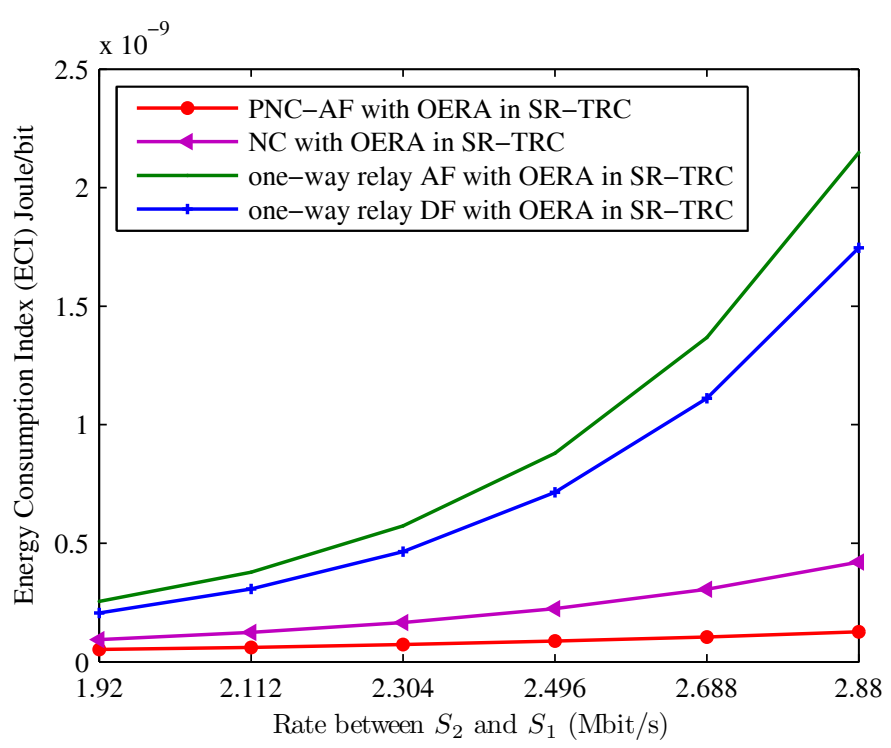

Figure 3 PNC gain with symmetric rate pair, where $\bar{r}_{1}=\bar{r}_{2}, K=1, W=15 \mathrm{kHz}, N=128, L=1000 \mathrm{~m}$.

number of bits (B) that were correctly delivered in the network during the same time period. For the two-way relay networks, ECI can be expressed as:

$$
E C I_{E / B}=\frac{E}{B}=\frac{\frac{1}{2} P_{\text {total }}}{\left(\bar{r}_{1}+\bar{r}_{2}\right)}[\text { Joule } / \text { bit }]
$$

where $P_{\text {total }}$ is the overall transmit power required to deliver required rates $\bar{r}_{1}$ and $\bar{r}_{2}$, and the factor $1 / 2$ comes from the two time slots required by messages exchange. We assume that the spectral density of noise, denoted by $N_{0}$, is equal to $-174 \mathrm{dBm} / \mathrm{Hz}$ and all the subcarrier complex gains are realized independently through complex normal distribution of the form $\mathcal{C N}\left(0, \frac{1}{\left(1+d_{i, j}\right)^{\Delta / 2}}\right)$, where $d_{i, j}$ is the distance between node $i$ and node $j$, and the path loss exponent $\Delta$ is 3 . We assume that $L$ is the distance between two users and all the relay nodes are randomly distributed between them.



Figure 4 PNC gain with asymmetric rate pair where $\bar{r}_{1}+\bar{r}_{2}=3.84 \mathrm{Mbit} / \mathrm{s}, K=1, W=15 \mathrm{kHz}, N=128, L=1000 \mathrm{~m}$. 
The ECI of each scheme is averaged over $1 \times 10^{8}$ independent realizations of relay' location by MonteCarlo simulation.

PNC gain is depicted in Figures 3 and 4, in which ECI of PNC-AF, NC, one-way relay AF and one-way relay DF with Optimal Energy-efficient Resource Allocation (OERA) are compared for TRC with single relay (SRTRC). ECI with symmetric rates denoted by $\bar{r}_{1}=\bar{r}_{2}$ is shown in Figure 3, which depicts that ECI increases with the sum-rate and that PNC-AF achieves PNC gain to greatly reduce the ECI compared with the other schemes. ECI with asymmetric rates is shown in Figure 4, where the $\mathrm{X}$-coordinate is the ratio of $\bar{r}_{1}$ to the fixed sum-rate with $\bar{r}_{1}+\bar{r}_{2}=3.84 \mathrm{Mbit} / \mathrm{s}$. Compared with other schemes, Figure 4 depicts PNC gain increases with the gap between $\bar{r}_{1}$ and $\bar{r}_{2}$ and minimum ECI is achieved while $\bar{r}_{1}=\bar{r}_{2}$.

Diversity gain is demonstrated in Figure 5, in which PNC-AF with OERA in SR-TRC and TRC with $K=$ $[2,5,20]$ relay nodes $(K R-T R C)$ are compared. The simulation depicts that PNC-AF with multiple relay nodes achieves great diversity gain which is directly proportional to the number of relay nodes, and the minimum ECI is achieved while $\bar{r}_{1}=\bar{r}_{2}$.

Figure 6 depicts the power allocation between $S_{1}, S_{2}$ and relay node(s). The X-coordinate is the ratio of 1000 to fixed sum-rate. Simulation shows that the average transmit power allocated to $S_{1}$ is directly proportional to the ratio of $\bar{r}_{1}$ to fixed sum-rate, while average transmit power allocated to $S_{2}$ is inversely proportional to the ratio. The transmit power of relay node(s) is minimum in symmetric rate pair condition, in which $\bar{r}_{1}=\bar{r}_{2}$.

\section{Conclusion and discussion}

In this article, we studied multi-user, multi-relay OFDMA-based two-way relay network with PNC-AF protocol. An optimal energy-efficient resource allocation with joint power allocation, relay selection and subcarrier assignment to achieve the minimum transmit power consumption with required link rate pair were derived. From the analysis, we proved that the optimal subcarrier assignment criterion in energy efficiency sense is an opportunistic subcarrier assignment, in which a subcarrier is assigned to a unique relay node. Based on the proof, the closed-form expressions of power allocation, relay selection, subcarrier assignment and minimum transmit power consumption were derived. The simulations confirmed the proposed scheme is far superior to the other existing schemes in terms of energy efficiency. It was also observed that energy efficiency of TRC is generally better with symmetric symmetric rate pair than asymmetric rate pair.

Beside the scenario considered in this article, energy efficiency can be further improved with more complex schemes, e.g., adaptive bit allocation on subcarriers and phase alignment operation at relay nodes. Furthermore, the analysis of energy efficiency is based on Shannon capacity expressions in this article, which do not take into account the impact of practical channel coding, modulation and retransmission methods. Hence, the

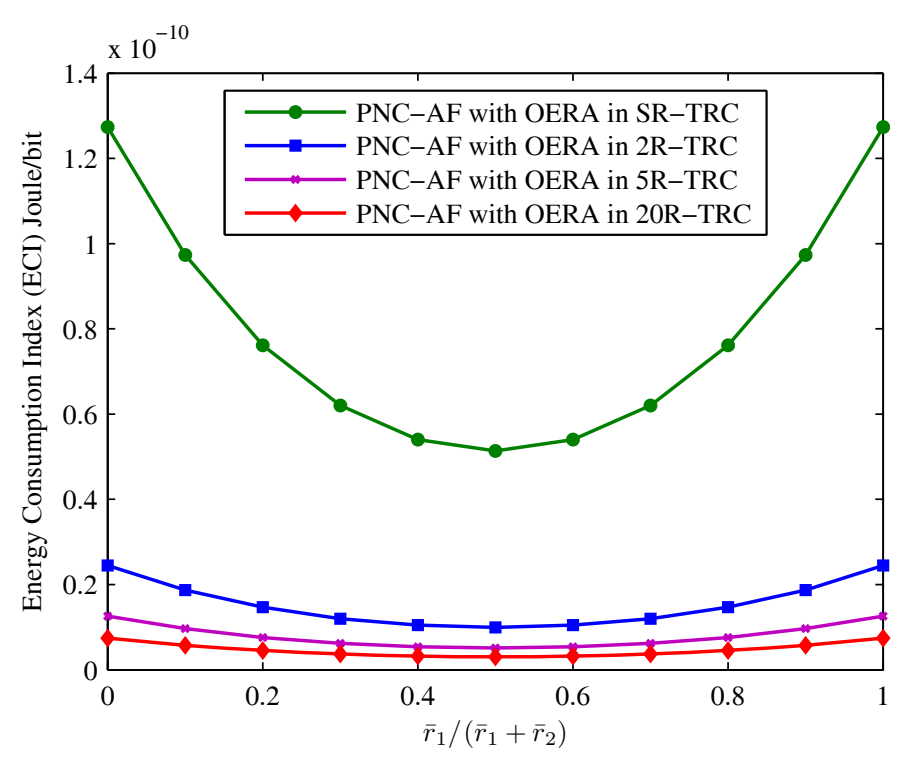

Figure 5 Diversity gain with asymmetric rate pair, where $\bar{r}_{1}+\bar{r}_{2}=3.84 \mathrm{Mbit} / \mathrm{s}, W=15 \mathrm{kHz}, N=128, L=1000 \mathrm{~m}$, and $K=1,2,5$, 20. 


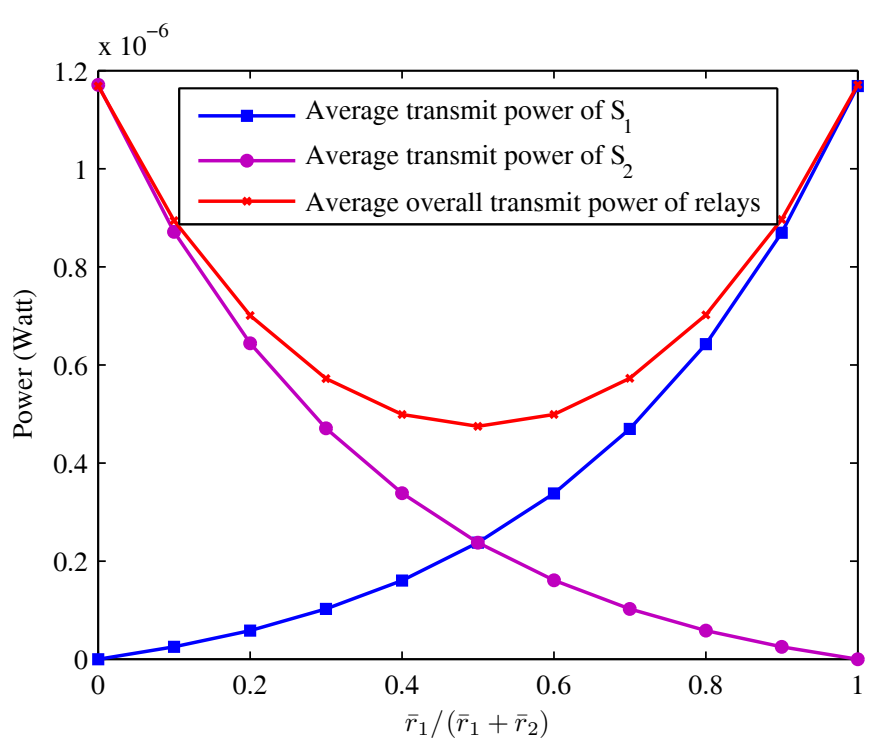

Figure 6 Power allocation between the users and relay node(s) with asymmetric rate pair, where $\bar{r}_{1}+\bar{r}_{2}=3.84 \mathrm{Mbit} / \mathrm{s}, W=15 \mathrm{kHz}$, $N=128, L=1000 \mathrm{~m}$.

consideration of energy efficiency in more practical scenarios will be another future research item.

\section{Acknowledgements}

This article is supported by International Scientific and Technological Cooperation Program (2010DFA11060), China-EU International Scientific and Technological Cooperation Program (0902), National Nature Science Foundation of China (Grant No. 61001119, 61027003) and Fund for Creative Research Groups of China (Grant No. 61121001). This article is partially sponsored by the Finnish Funding Agency for Technology and Innovation (Tekes), under the project Energy and Cost Efficiency for Wireless Access.

\section{Author details}

${ }^{1}$ Wireless Technology Innovation Institute, Key Laboratory of Universal Wireless Communication, Ministry of Education, Beijing University of Posts and Telecommunications, Beijing, 100876, China ${ }^{2}$ Department of Communications Engineering, Tampere University of Technology, Tampere, P.O.Box 553, Fl-33101, Finland

\section{Competing interests}

The authors declare that they have no competing interests.

Received: 19 July 2011 Accepted: 27 February 2012

Published: 27 February 2012

\section{References}

1. S Parkvall, E Dahlman, A Furuskar, Y Jading, M Olsson, S Wanstedt, K Zangi, LTE-Advanced - Evolving LTE towards IMT-Advanced, in IEEE 68th Vehicular Technology Conference, 2008. VTC 2008-Fall, Calgary, Canada, 1-5 (2008)

2. SW Peters, AY Panah, KT Truong, RW Heath, Relay architectures for 3GPP LTE-Advanced. EURASIP J Wirel Commun Netw. 2009 (2009)

3. S Zhang, SC Liew, P PLam, Hot topic: physical layer network coding, in Proc International conference on Mobile computing and networking (MobiCom'06), Los Angeles, USA, 358-365 (2006)

4. P Popovski, H Yomo, Physical network coding in two-way wireless relay channels, in IEEE International Conference on Communications, Glasgow, Scotland, 707-712 (2007)
5. P Popovski, H Yomo, The anti-packets can increase the achievable throughput of a wireless multi-hop network, in IEEE International Conference on Communications, 2006. 9, 3885-3890 (2006)

6. S Katti, S Gollakota, D Katabi, Embracing wireless interference: analog network coding, in ACM SIGCOMM 2007, Kyoto, Japan, 9, 397-408 (2007)

7. R Louie, Y Li, B Vucetic, Practical physical layer network coding for two-way relay channels: performance analysis and comparison. IEEE Trans Wirel Commun. 9(2), 764-777 (2010)

8. Y Wu, PA Chou, SY Kung, Information exchange in wireless networks with network coding and physical-layer broadcast, Tech Rep MSR-TR-2004-78, Microsoft Research, Redmond, Wash, USA, 2004

9. CK Ho, R Zhang, YC Liang, Two-way relaying over OFDM: optimized tone permutation and power allocation, in IEEE International Conference on Communications, 2008. ICC'08, 3908-392 (2008)

10. M Dong, S Shahbazpanahi, Optimal spectrum sharing and power allocation for OFDM-based two-way relaying, in 2010 IEEE International Conference on Acoustics Speech and Signal Processing (ICASSP), 3310-3313 (2010)

11. YU Jang, ER Jeong, Y Lee, A two-step approach to power allocation for OFDM signals over two-way amplify-and-forward relay. IEEE Trans Signal Process. 58(4), 2426-2430 (2010)

12. K Jitvanichphaibool, R Zhang, YC Liang, Optimal resource allocation for two-way relay assisted OFDMA. IEEE Trans Veh Technol. 58(7), 3311-3321 (2009)

13. H Shin, JH Lee, Subcarrier allocation for multiuser two-way OFDMA relay networks with fairness constraints, in 2010 IEEE 71st Vehicular Technology Conference, 1-5 (2010)

14. LM Correia, D Zeller, B Oliver, D Ferling, Y Jading, I Godor, G Auer, L Van Der Perre, Challenges and enabling technologies for energy aware mobile radio networks. IEEE Commun Mag. 48, 66-72 (2010)

15. D2.3 - Energy efficiency analysis of the reference systems, areas of improvements and target breakdown. EARTH project, 39-40, (2010), https:// bscw.ict-earth.eu/pub/bscw.cgi/d31515/EARTH_WP2_D2.3.pdf

16. S Boyd, L Vandenberghe, Convex Optimization (Cambridge University Press, Cambridge, UK, 2004)

17. JL Troutman, Variational Calculus and Optimal Control: Optimization with Elementary Convexity (Springer, US, 1995)

18. DG Luenberger, Y Ye, Linear and Nonlinear Programming (Springer, US, 2009)

19. RA Horn, CR Johnson, Matrix Analysis (Cambridge University Press, UK, 1985) 
20. B Luo, Q Cui, H Wang, X Tao, Optimal joint water-filling for coordinated transmission over frequency-selective fading channels. IEEE Commun Lett. 15(2), 190-192 (2011)

doi:10.1186/1687-1499-2012-66

Cite this article as: Zhou et al: Energy-efficient resource allocation for OFDMA-based two-way relay channel with physical-layer network coding. EURASIP Journal on Wireless Communications and Networking 2012 2012:66.

\section{Submit your manuscript to a SpringerOpen ${ }^{\circ}$ journal and benefit from:}

- Convenient online submission

- Rigorous peer review

- Immediate publication on acceptance

- Open access: articles freely available online

- High visibility within the field

- Retaining the copyright to your article

Submit your next manuscript at $\gg$ springeropen.com 